\title{
Are Bismuth Shields Useful in Dentomaxillofacial Radiology Practice for the Protection of Eyes and Thyroid Glands from Ionizing Radiation?
}

\author{
Emre Aytugar,, ${ }^{1,}$ Taha Emre Kose, ${ }^{2}$ Birsay Gumru, ${ }^{3}$ Tugce Bicer Aytugar, ${ }^{4}$ Dogan Yasar, ${ }^{5}$ Erhan Cene, ${ }^{6}$ \\ and Ahmet Mihmanli ${ }^{7}$ \\ ${ }^{1}$ Department of Oral and Maxillofacial Radiology, Faculty of Dentistry, Izmir Katip Celebi University, Izmir, Turkey \\ ${ }^{2}$ Department of Oral and Maxillofacial Radiology, Faculty of Dentistry, Istanbul University, Istanbul, Turkey \\ ${ }^{3}$ Department of Oral and Maxillofacial Radiology, Faculty of Dentistry, Marmara University, Istanbul, Turkey \\ ${ }^{4}$ Department of Oral and Maxillofacial Surgery, Faculty of Dentistry, Izmir Katip Celebi University, Izmir, Turkey \\ ${ }^{5}$ Secondary Standard Dosimetry Laboratory, Cekmece Nuclear Research and Training Center, Turkish Atomic Energy Authority, Istanbul, Turkey \\ ${ }^{6}$ Department of Statistics, Yildiz Technical University, Istanbul, Turkey \\ ${ }^{7}$ Department of Oral and Maxillofacial Surgery, Faculty of Dentistry, Bezmialem Vakif University, Istanbul, Turkey \\ "Corresponding author: Emre Aytugar, Department of Oral and Maxillofacial Radiology, Faculty of Dentistry, Izmir Katip Celebi University, Aydinlik Evler Quarter, Cemil Meric \\ Avenue, 6780 Street, No:48, 35640, Cigli, Izmir, Turkey. Tel: +90-2323254040, E-mail: emreaytugar@gmail.com
}

Received 2016 July 20; Revised 2017 January 16; Accepted 2017 January 25.

\begin{abstract}
Background: The eye lens and thyroid gland are sensitive to radiation and have a risk of being exposed to primary beams and scattered radiation during dental radiographic examinations.

Objectives: The present study was aimed to evaluate the effectiveness of bismuth shielding in decreasing eye lens and thyroid radiation dose in periapical radiography (PR), orthopantomography (OPTG), and cone beam computed tomography (CBCT) examinations. There is no information in relevant literature regarding the use of bismuth shielding in dental radiology.

Materials and Methods: Dose measurements for PR, OPTG and CBCT were conducted by placing thermoluminescent dosimeter chips on the eye and thyroid location in a standard head phantom. Each one of the scan techniques was performed without any bismuth shielding, and they were then repeated with bismuth shielding in place. The percent dose reduction from bismuth shielding was calculated by dividing the dose measured with bismuth shielding by the dose measured without bismuth shielding.

Results: The eye dose was observed to decrease in bismuth shielding group in PR, however it was not statistically significant. The thyroid dose was found to be significantly decreased in PR $(\mathrm{P}<0.05)$. In OPTG and CBCT scans, both the dose to the eye and the thyroid gland was observed to increase by using bismuth shielding. Among these, only the increase in the eye dose in CBCT was not statistically significant $(\mathrm{P}>0.05)$.

Conclusion: This study demonstrated higher thyroid and eye lens dose with the use of bismuth shielding in OPTG and CBCT scanning. However, due to the significantly decreased thyroid dose in PR, it may be recommended as an alternative to leaded shielding in periapical radiographic examinations.
\end{abstract}

Keywords: Periapical Radiography, Panoramic Radiography, Cone Beam Computed Tomography, Bismuth Shielding, Dose Reduction

\section{Background}

Despite being one of the most commonly used assisting examination methods in dentistry, the radiation doses in dental radiographic practices are relatively low. The dose for a periapical radiograph (PR) is equal to the background radiation a patient will receive in only a few days and is considered to increase the risk of cancer less than 1 in 1 million. However, in computed tomography(CT) scans, depending on the size of scanned area and scanning pa- rameters, the dose received by the patient equals to 2 3 years of background radiation, and is considered to increase the risk of cancer by approximately 1 in 1000 and 1 in 10.000 (1).

In dentistry, orthopantomography (OPTG) is a routine diagnostic tool that offers a relatively low dose of radiation. However, OPTG is used more frequently than other methods of radiographic examination, and it primarily irradiates the head and the neck, where the lenses of the eyes, salivary glands, and the thyroid gland are located. 
Therefore, it is important to evaluate and minimize the effective dose of OPTG (2).

Cone beam computed tomography (CBCT), which has become an established modality in dentomaxillofacial imaging in recent years with the advantage of providing three-dimensional and high-quality images of particularly the bone tissue (1).

The eye lens and thyroid gland, which are sensitive to radiation, have a risk of being exposed to primary beams and scattered radiation during dental radiographic examinations (3). The International Commission on Radiological Protection (ICRP) (4) has scrutinized recent epidemiological evidence suggesting that the threshold in the absorbed dose for cataractogenesis is now considered to be as low as $0.5 \mathrm{~Gy}$ for the lens of the eye. It is considered that the thyroid gland dose is low in dental applications (3); however, it should be noted that exposure to any dose of radiation may lead to biological damage (5). According to the Biologic Effects of Ionizing Radiation (BEIR) report VII (6), thyroid gland is particularly radiosensitive with stochastic effects that specifically include the induction of thyroid carcinoma.

As the risk in patients younger than 10 years is three times greater in comparison to adults at the age of 30, it is critical to adopt strategies to ensure the optimization of the radiation dose to such radiosensitive organs in pediatric population and young adults who are known to be more sensitive to radiation $(5,7,8)$. Therefore, the diagnostic radiographic doses in dentistry should be kept As Low As Reasonably Achievable (ALARA) while still producing reasonable image quality, and radiosensitive organs should be protected.

Reducing tube current time product (mAs), using tube current modulation, reducing peak-voltage $(\mathrm{kVp})$, using relatively higher pitches, and limiting both scan regions and multiphase examinations are among the methods that are used to reduce the radiation dose for CT (9-11). Although it is possible to reduce the $\mathrm{mA}$ and $\mathrm{kVp}$ parameters in OPTG devices, PR devices generally do not permit a change in the $\mathrm{kVp}$. There are various disadvantages of all these mentioned methods.

In addition, the use of thyroid collars, leaded aprons, leaded glasses and bismuth shielding in order to shield radiosensitive organs in the region of scanning has been advocated (10). The National Council on Radiation Protection and Measurements (NCRP) (12) recommends the use of thyroid shielding for children and indicates that thyroid shielding should be used for adults as long as it will not interfere with the examination. Recently, the American Thyroid Association (ATA) (13) issued new guidelines on how to minimize any unnecessary exposure to radiation during the execution of medical and dental imaging procedures, such as the use of thyroid collars for dental X-rays. Thyroid collars are used in intraoral and cephalometric examinations; however, they are not used in OPTG due to the fact that they may prevent the primary beam, and their use in CBCT requires further inquiry (14).

The shields made of bismuth-impregnated latex for specific organs in CT scans were first utilized in the 1990 s (11). Elastic, form-fitting, inert bismuth shields are designed to be placed over an organ of interest and can provide a meaningful reduction of dose as indicated in a number of studies combined with their ease of use $(11,15,16)$. Bismuth shielding is commercially available in different thicknesses and bismuth shielding of $1 \mathrm{~mm}$ thickness is considered to have lead equivalent of $0.06 \mathrm{~mm}(9,17)$. As opposed to the leaded garments that completely block the $\mathrm{X}$-ray from the patient, bismuth shielding partially block the X-ray beam by removing the low-energy photons that would deliver radiation but would not contribute in image formation to reduce the dose to the underlying tissue while allowing enough X-rays to pass in order to enable diagnostic image formation. Thus, a reduction of dose without loss in image quality is provided (16).

\section{Objectives}

To the best of our knowledge, there are no studies that evaluate the effectiveness of bismuth shielding in dental radiographic examinations. Thus, the aim of this study was to evaluate the effectiveness of bismuth shielding in decreasing eye and thyroid radiation dose in PR, OPTG, and CBCT examinations.

\section{Materials and Methods}

\subsection{Devices, Parameters, Phantom, Absorbed Dose Measure-} ments

This study was conducted in the department of oral and maxillofacial radiology, faculty of dentistry, Bezmialem University, Istanbul, Turkey.

The equipment used was a periapical X-ray device (Belmont 303-H, 60/70 kVp, 4/7 mA, Takara Belmont Corp., Osaka, Japan), a panoramic imaging device (Planmeca Promax, Helsinki, Finland), and a CBCT scanner (Planmeca ProMax 3D Mid, Helsinki, Finland). Exposure parameters for PR, OPTG and CBCT were set at 60/70 kVp, 4/7 mA, and 0.2 seconds; $64 \mathrm{kVp}, 6 \mathrm{~mA}$, and 16 seconds; and $90 \mathrm{kVp}, 10 \mathrm{~mA}$, and 18 seconds, respectively. Field of view (FOV) area of 160 $\mathrm{mm}$ diameter by $90 \mathrm{~mm}$ height was used in CBCT scans.

All exposures were performed with aforementioned commercially available dental devices by using selected collimation and exposure techniques. Using a standard 
head phantom (P-6/3 TSE Premium Head with Torso Assembly, Frasaco, USA), each of the three types of scans were repeated three times with thermoluminescent dosimeters (TLDs) placed over the eye, first without any bismuth shielding, and then repeated three times with bismuth eye shields (AttenuRad Radiation Protection; F\&L Medical Co., Vandergrift, PA; $0.060 \mathrm{~mm} \mathrm{~Pb}$ equivalent) in place. The same procedure was repeated for the thyroid gland using bismuth thyroid shields (AttenuRad Radiation Protection; F\&L Medical Co., Vandergrift, PA; 0.060 mm Pb equivalent). These shields, which are commercially available in three different shapes for the breast, the eye lens and the thyroid, are made up of four layers of bismuth radioprotective latex, each layer containing $0.0085 \mathrm{~g} / \mathrm{cm}^{2}$ of bismuth, which is equivalent to $0.015 \mathrm{~mm}$ of lead $(\mathrm{Pb})[14 \%$ attenuation at $120 \mathrm{kV}$ half-value layer (HVL) $5.6 \mathrm{mmAl}]$.

Dose measurements were conducted by placing TLDs on the phantom eye and thyroid location. For each scan, three TLDs were initially placed next to each other over the center of the eye or the thyroid gland. After exposures for each scan type was completed, the TLDs were removed from the phantom and placed into a storage container. TLDs that were unexposed were then placed in the exact same locations on the phantom, and the next exposure technique was performed. Special attention was paid to always place the TLD in the same orientation for each type of scan.

Exposed TLDs were transported with unexposed TLDs to serve as negative controls (background readings). All dosimeters were read three times, and background readings were subtracted from the averaged readings. All dosimeters were read, and background readings were subtracted from the readings.

\subsection{Thermoluminescent Dosimeter Specifications}

The TLDs used in this study (TLD-100H, Harshaw Lithium fluoride (LiF) TLD-100H, Saint-Gobain Industrial Ceramics, Solon, OH, USA) were lithium fluoride (LiF) square shaped chips (side $3 \mathrm{~mm}$, thickness $0.8 \mathrm{~mm}$ ) doped with magnesium $(\mathrm{Mg})$, copper $(\mathrm{Cu})$, and phosphorus $(\mathrm{P})$. Calibration and analysis of the TLDs were performed by the Turkish atomic energy authority, Cekmece nuclear research and training center, secondary standard dosimetry laboratory (Istanbul, Turkey). Before use, the TLDs were annealed at $240^{\circ} \mathrm{C}$ for 10 minutes and then immediately cooled to ambient temperature to eliminate any residual light. A Harshaw 4500 TLD reader was used to enable the reading of the TLDs. The reading cycle of a chip commenced with a preheating period (reaching $50^{\circ} \mathrm{C}$ in $13 \mathrm{sec}-$ onds), followed by reading of the air kerma at $25^{\circ} \mathrm{C} / \mathrm{s}$ up to $300^{\circ} \mathrm{C}$. Following every ten readings, the stability of the
TLD reader was checked by using the reference light source available in the reader.

Reference dose measurement was achieved by a working standard in which the calibration factor was traceable to the Physikalisch-Technische Bundesanstalt. Prior to the examinations, the variation in sensitivity of the TLD chips (element correction coefficient) was investigated. The chips were exposed to radiation in the $137 \mathrm{Cs}$ radioactive source at the Secondary standards dosimetry laboratory, giving an air kerma value of $5 \mathrm{mGy}$. Individual calibration factors (ECC, element correction coefficient) were thus determined for each chip. The sensitivity of each chip in comparison to the average sensitivity of the entire batch was obtained and used in all further measurements. The reader calibration factor (RCF) was determined from golden chips irradiated at RQR7 quality, which is described as an X-ray tube voltage of $90 \mathrm{kVp}$ for diagnostic radiation quality.

\subsection{Statistical Analysis}

Means and standard deviations were calculated using IBM SPSS statistics software 22.0 (IBM Co., Armonk, NY, USA). Doses resulting from each protocol were assessed statistically using Wilcoxon signed-rank test. Significance was set at $\mathrm{P}<0.05$. The percent reduction from bismuth shielding was calculated by dividing the dose measured with bismuth shielding by the dose measured without bismuth shielding.

\section{Results}

The results of the study are provided in Table 1 . However it was not statistically significant, the eye dose in bismuth shielding group in PR was lower. The thyroid dose was found to be significantly lower in bismuth shielding group in $\mathrm{PR}(\mathrm{P}<0.05)$.

In OPTG and CBCT scans, both the dose to the eye and the thyroid gland was observed to be higher by using bismuth shielding. Among these, only the eye dose in CBCT was not statistically significant $(\mathrm{P}>0.05)$.

\section{Discussion}

In dental practice, lead shielding has an important place to protect the thyroid gland and lens, and its effectiveness has been demonstrated by several studies $(7,18$, 19). Hoogeveen et al. (19) reported a dose reduction of $75 \%$ in the periapical exposures of upper anterior region using thyroid shielding and concluded that thyroid shielding results in significant thyroid dose reduction not only in the upper anterior region, but also in radiography of all upper 


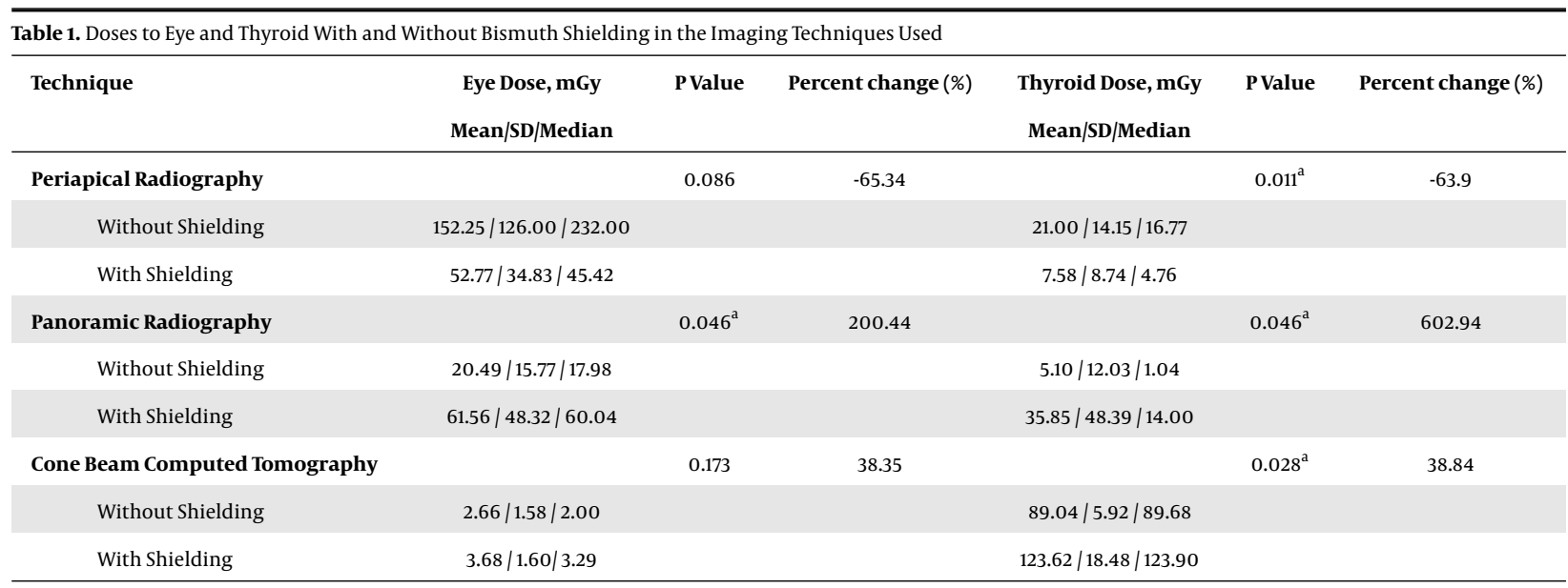

Wilcoxon signed-rank test; ${ }^{\mathrm{a}} \mathrm{P}<0.05$, statistically significant.

teeth. Qu et al. (7), who studied the effectiveness of thyroid collar shielding in different FOVs in CBCT scanning, found a reduction of $18 \%-40.1 \%$ with a single thyroid collar. Given that the image quality of the mandible may be affected by the use of thyroid collars, they recommended that patients might be asked to lift their chin so that the inferior border of the mandible lies parallel to the horizontal plane during CBCT scanning. Effectiveness of lead shielding was also shown in studies regarding lead glasses. Prins et al. (18) reported a decrease of $62 \%$ for full FOV and $36 \%$ for collimated scan in the radiation dose to the eye.

Although lead thyroid collars and lead glasses are proved to be effective in dose reduction, large lead glasses decrease image quality of the area between the maxillary sinus floor and the orbit, and thyroid shielding impairs the image quality in OPTG and CBCT scanning $(7,14,20)$. Therefore, bismuth shielding may be considered as an alternative in shielding these areas. Bismuth shielding has been proven to be effective in dose reduction in the breast, thyroid gland and optic lens. However, to our knowledge there are no studies regarding the effectiveness of bismuth shielding in dental practice.

In PR of especially upper anterior teeth, thyroid gland is subjected to primary beams. The dose received by this area is shown to be considerably reduced with the use of thyroid shielding (19). In this study, dose reduction in the thyroid region during the PR of upper anterior teeth obtained with bismuth shielding (63.90\%) was lower in comparison to that obtained with lead shielding by Hoogeveen et al. (75\%) (19). This difference may be explained by the fact that bismuth shielding partially blocks the X-ray beam to the relevant area removing the low-energy photons. Despite providing lower dose reduction compared to lead collars, dose reduction over $60 \%$ should not be underesti- mated and bismuth shielding still offers a good option for protection of thyroid in intraoral radiography.

OPTG covers the lower $1 / 3$ of the orbital region, and the thyroid gland and optic lens are exposed to radiation in OPTG imaging $(2,21)$. The use of bismuth shielding resulted in an increase in dose (200.44\%) in the eye region in this study. As in OPTG scanning, to display the anterior region the irradiation is performed while the tube is in the posterior aspect, and in order for beams to pass through dense areas such as the spine and skull base the dose is high, these beams may be creating backscatter radiation on the bismuth shield falling back on the eye. The increase in the thyroid dose in this study (602.94\%) in OPTG scanning with the use of bismuth shielding may be explained by backscatter radiation as well. Han et al. (22) reported a 9.6-22.7\% reduction in thyroid dose with the use of leaded shielding in OPTG scanning. However, no studies have been conducted using bismuth shielding. Strong beams may also pass through the bismuth shielding after passing through the patient, but as the OPTG device operates at $64 \mathrm{kVp}$, the beam that passes through the patient gets stuck and reflected back from bismuth shielding. In addition, X-ray beam is sent from posterior aspect in order to get the image of anterior region in OPTG devices, and the bismuth shield will hold the beam passing through patient in order to produce image on the detector. Therefore the use of bismuth shielding in OPTG imaging will also cause problems in terms of image quality.

In several studies, reduction in radiation doses to the thyroid and the eye with bismuth shielding was shown regarding CT operating at $120 \mathrm{kVp}(11,23,24)$. Inkoom et al. (25) investigated the effect of bismuth shielding on thyroid dose and image quality in CT with fixed exposure parameters using pediatric anthropomorphic phantoms repre- 
senting the equivalent newborn and 10-year-old child and reported a $17 \%$ and $35 \%$ decrease in thyroid dose, respectively. They also reported that the placement of a cotton spacer between the bismuth shield and the tissue was observed to have no significant effect on the thyroid radiation dose. In our study, increase in thyroid and eye dose with the use of bismuth shielding was observed in CBCT scanning. This may be a result of different exposure parameters used and difference in the beam collimation in $\mathrm{CT}$ and СBCT. In their study regarding the use of bismuth shielding in CT, Kim et al. (26) reported that despite the increased thyroid dose due to backscatter, the radiation dose was reduced in general, and the dose increase from backscatter varying due to different parameters used (between 0.7 and $2.6 \%$ ) was found to be negligible. In our study, increase in thyroid and eye dose with the use of bismuth shielding was observed in CBCT scanning. This may be a result of different exposure parameters used and difference in the beam collimation in CT and CBCT.

There are some limitations to this study. The results of this study should be cautiously translated to the clinical situation, as this is a laboratory-based research study. To the authors' knowledge, this is the first study evaluating the effectiveness of bismuth shielding in decreasing eye and thyroid dose in PR, OPTG, and CBCT examinations. Therefore, direct comparison with other studies was not possible. The use of such type of shielding is the object of considerable debate, and there are controversies with respect to its practical application (15). The potential increase in image noise, beam hardening and scatter artifacts, and decrease in image quality are the factors that hinder the widespread adoption of bismuth shielding $(9,10)$. Some researchers blame the occurrence of streak artefacts on the improper application of bismuth shielding $(10,16)$. Any air spaces or wrinkles in the shielding substance will result in significant beam hardening artifact. In addition to these findings, there are findings regarding decreased dosage without loss of image quality $(11,23)$. In 2012, The American association of physicists in medicine (AAPM) (27) published its stance on the use of bismuth shields to achieve dose reduction and recommended that alternative methods should be considered and applied whenever possible. The reasons for this were problems that might occur in automatic exposure control (AEC) usage, causing attenuation in beams from patient to get imaging where the beam is given from the posterior (since 3600 irradiation is done with CT) (since $360^{\circ}$ irradiation is done with CT) and increased noise caused by this.

Another limitation is that a standard head phantom was used in this study because an anthropometric one was not available. In a study by Hopper et al. (28), the ability of bismuth in reducing radiation to the lens of the eye during routine cranial CT examination was tested conducting both phantom and human studies. Phantom studies were performed using a standard head phantom as in our study, and demonstrated similar results to the patient study. The authors concluded that bismuth-coated latex shielding of the eye during cranial CT examination is simple to apply, inexpensive, and causes up to a 50\% reduction in radiation to the lens of the eye. Our study should be considered as an initial study to attract attention on the use of bismuth shielding in dental radiology and should be supported by future studies done by using anthropometric phantoms. Another limitation is that a standard head phantom was used in this study because an anthropometric one was not available. In addition, although the use of TLDs in dosimetry phantoms is a common methodology used in dose studies, there is a lack of consensus regarding the position and number of TLDs in a phantom, and variations were recorded even when the same phantom was used. A wide range of TLDs has been used to record the dose to the thyroid gland, ranging from one to seven TLDs. There is a general agreement that the use of several TLDs for each organ allows for a more accurate average organ dose to be calculated. As the present study focused on the eye and thyroid gland dose, the use of three TLDs in these regions was considered most practicable.

In conclusion, there is no information in relevant literature regarding the use of bismuth shielding in dental radiology. This study demonstrated increase in thyroid and eye dose in OPTG and CBCT imaging, and decrease in thyroid dose in PR with the use of bismuth shielding. Future studies are necessary for bismuth shields to be considered as an alternative to lead shielding in PR.

\section{Acknowledgments}

We want to thank Assist. Prof. Omer Uysal from Bezmialem Vakif University, Faculty of Medicine, Department of Biostatistics and Medical Informatics for statistical analysis.

\section{References}

1. Al Najjar A, Colosi D, Dauer LT, Prins R, Patchell G, Branets I, et al. Comparison of adult and child radiation equivalent doses from 2 dental cone-beam computed tomography units. Am J Orthod Dentofacial Orthop. 2013;143(6):784-92. doi: 10.1016/j.ajodo.2013.01.013. [PubMed: 23726328].

2. Lee C, Lee SS, Kim JE, Huh KH, Yi WJ, Heo MS, et al. Comparison of dosimetry methods for panoramic radiography: thermoluminescent dosimeter measurement versus personal computer-based Monte Carlo method calculation. Oral Surg Oral Med Oral Pathol Oral Radiol. 2016;121(3):322-9. doi: 10.1016/j.oooo.2015.10.030. [PubMed: 26795453]. 
3. Rush ER, Thompson NA. Dental radiography technique and equipment: How they influence the radiation dose received at the level of the thyroid gland. Radiography. 2007;13(3):214-20. doi: 10.1016/j.radi.2006.03.002.

4. Authors on behalf of I, Stewart FA, Akleyev AV, Hauer-Jensen M, Hendry JH, Kleiman NJ, et al. ICRP publication 118: ICRP statement on tissue reactions and early and late effects of radiation in normal tissues and organs-threshold doses for tissue reactions in a radiation protection context. Ann ICRP. 2012;41(1-2):1-322. doi: 10.1016/j.icrp.2012.02.001. [PubMed: 22925378].

5. Cordasco G, Portelli M, Militi A, Nucera R, Lo Giudice A, Gatto E, et al. Low-dose protocol of the spiral CT in orthodontics: comparative evaluation of entrance skin dose with traditional X-ray techniques. Prog Orthod. 2013;14:24. doi: 10.1186/2196-1042-14-24. [PubMed: 24325970].

6. National Research Council. Health risks from exposure to low levels of ionizing radiation: BEIR VII phase 2. 7. National Academies Press; 2006.

7. Qu X, Li G, Zhang Z, Ma X. Thyroid shields for radiation dose reduction during cone beam computed tomography scanning for different oral and maxillofacial regions. Eur JRadiol.2012;81(3):e376-80. doi: 10.1016/j.ejrad.2011.11.048. [PubMed: 22209523].

8. Shin HS, Nam KC, Park H, Choi HU, Kim HY, Park CS. Effective doses from panoramic radiography and CBCT (cone beam CT) using dose area product (DAP) in dentistry. Dentomaxillofac Radiol. 2014;43(5):20130439. doi: 10.1259/dmfr.20130439. [PubMed: 24845340].

9. Vollmar SV, Kalender WA. Reduction of dose to the female breast in thoracic CT: a comparison of standard-protocol, bismuth-shielded, partial and tube-current-modulated CT examinations. Eur Radiol. 2008;18(8):1674-82. doi: 10.1007/s00330-008-0934-9. [PubMed: 18414873].

10. Kim S, Frush DP, Yoshizumi TT. Bismuth shielding in CT: support for use in children. Pediatr Radiol. 2010;40(11):1739-43. doi: 10.1007/s00247-010-1807-3. [PubMed: 20734038].

11. Mendes M, Costa F, Figueira C, Madeira P, Teles P, Vaz P. Assessment of patient dose reduction by bismuth shielding in CT using measurements, GEANT4 and MCNPX simulations. Radiat Prot Dosimetry. 2015;165(1-4):175-81. doi: 10.1093/rpd/ncv059. [PubMed: 25813483].

12. National Council on Radiation Protection and Measurements. Radiation protection in dentistry: recommendations of the National Council on Radiation Protection and Measurements. Bethesda: National Council on Radiation Protection and Measurements; 2003.

13. American Thyroid Association (ATA). Policy Statement on Thyroid Shielding During Diagnostic Medical and Dental Radiology. 2016. Available from: http://www.thyroid.org/wp-content/uploads/statements/ ABS1223_policy_statement.pdf.

14. Kelaranta A, Ekholm M, Toroi P, Kortesniemi M. Radiation exposure to foetus and breasts from dental X-ray examinations: effect of lead shields. Dentomaxillofac Radiol. 2016;45(1):20150095. doi: 10.1259/dmfr.20150095. [PubMed: 26313308].

15. Correia PD, Granzotti CR, Santos Yda S, Brochi MA, de AzevedoMarques PM. Characterization of a lead breast shielding for dose reduction in computed tomography. Radiol Bras. 2014;47(4):223-7. doi: 10.1590/0100-3984.2013.1861. [PubMed: 25741089].
16. Samei E. Pros and cons of organ shielding for CT imaging. Pediatr Radiol. 2014;44 Suppl 3:495-500. doi: 10.1007/s00247-014-3084-Z. [PubMed: 25304710].

17. Einstein AJ, Elliston CD, Groves DW, Cheng B, Wolff SD, Pearson GD et al. Effect of bismuth breast shielding on radiation dose and image quality in coronary CT angiography. J Nucl Cardiol. 2012;19(1):100-8. doi: 10.1007/s12350-011-9473-x. [PubMed: 22068687]

18. Prins R, Dauer LT, Colosi DC, Quinn B, Kleiman NJ, Bohle GC, et al. Significant reduction in dental cone beam computed tomography (CBCT) eye dose through the use of leaded glasses. Oral Surg Oral Med Oral Pathol Oral Radiol Endod. 2011;112(4):502-7. doi: 10.1016/j.tripleo.2011.04.041. [PubMed: 21802322].

19. Hoogeveen RC, Hazenoot B, Sanderink GC, Berkhout WE. The value of thyroid shielding in intraoral radiography. Dentomaxillofac Radiol. 2016;45(5):20150407. doi: 10.1259/dmfr.20150407. [PubMed: 27008105].

20. Goren AD, Prins RD, Dauer LT, Quinn B, Al-Najjar A, Faber RD, et al. Effect of leaded glasses and thyroid shielding on cone beam CT radiation dose in an adult female phantom. Dentomaxillofac Radiol. 2013;42(6):20120260. doi: 10.1259/dmfr.20120260. [PubMed: 23412460].

21. Akyalcin S, English JD, Abramovitch KM, Rong XJ. Measurement of skin dose from cone-beam computed tomography imaging. Head Face Med. 2013;9:28. doi: 10.1186/1746-160X-9-28. [PubMed: 24192155].

22. Han GS, Cheng JG, Li G, Ma XC. Shielding effect of thyroid collar for digital panoramic radiography. Dentomaxillofac Radiol. 2013;42(9):20130265. doi: 10.1259/dmfr.20130265. [PubMed: 24005060].

23. Catuzzo P, Aimonetto S, Fanelli G, Marchisio P, Meloni T, Mistretta L, et al. Dose reduction in multislice CT by means of bismuth shields: results of in vivo measurements and computed evaluation. Radiol Med. 2010;115(1):152-69. doi: 10.1007/s11547-009-0469-4. [PubMed: 20012921].

24. Chang KH, Lee W, Choo DM, Lee CS, Kim Y. Dose reduction in CT using bismuth shielding: measurements and Monte Carlo simulations. Radiat Prot Dosimetry. 2010;138(4):382-8. doi: 10.1093/rpd/ncp278. [PubMed: 19959602].

25. Inkoom S, Papadakis AE, Raissaki M, Perisinakis K, Schandorf C, Fletcher JJ, et al. Paediatric Neck Multidetector Computed Tomography: The Effect of Bismuth Shielding on Thyroid Dose and Image Quality. Radiat Prot Dosimetry. 2016. doi: 10.1093/rpd/ncw007. [PubMed: 26891787].

26. Kim S, Yoshizumi TT, Frush DP, Anderson-Evans C, Toncheva G. Dosimetric characterisation of bismuth shields in CT: measurements and Monte Carlo simulations. Radiat Prot Dosimetry. 2009;133(2):105-10. doi: 10.1093/rpd/ncp025. [PubMed: 19264831].

27. American Association of Physicists in Medicine (AAPM). Position Statement on the Use of Bismuth Shielding for the Purpose of Dose Reduction in CT Scanning. 2016. Available from: https://www.aapm.org/ publicgeneral/BismuthShielding.pdf.

28. Hopper KD, Neuman JD, King SH, Kunselman AR. Radioprotection to the eye during CT scanning. AJNR Am J Neuroradiol. 2001;22(6):1194-8. [PubMed: 11415918]. 